\title{
Real Time Design and Realization of Digital Radar Pulse Compression Based on Linear Frequency Modulation (LFM) without Components around Zero Frequency Using FPGA
}

\author{
H. A. Said ${ }^{1}$, A. A. El-Kouny ${ }^{2}$, A. E. El-Henawey ${ }^{1}$ \\ ${ }^{1}$ Faculity of Engineering, Ain Shams University \\ ${ }^{2}$ Misr University of Science and Technology
}

\begin{abstract}
Good range resolution can be achieved with a shorter pulse. But on the other hand, shorter pulses need more peak power. The shorter the pulse gets, the more should its peak power be increased so that enough energy is packed into the pulse. High Peak Power makes the design of transmitters and receivers more difficult since the components used to construct these must be able to withstand the peak power. One way to overcome the problem of high peak power is to convert the short pulse into a long one and using some form of modulation to increase the bandwidth of the long pulse so that the range resolution is not compromised. This technique is called Pulse Compression and is used widely in Radar applications where high peak power is undesirable. This paper introduces a new radar pulse compression system, using a type of Linear Frequency Modulation (LFM) signal which lacks components around zero frequency. A core structure of a pulse processor is similar to a standard matched filter, modified to suit the application. Flexibility in the design permits the use of multiple code types and lengths needed, which eliminate ambiguities, caused by high pulse repetition frequencies. The device used in this application is a field programmable gate array (FPGA) which has distinct advantages compared to other application specific integrated circuits (ASIC) for the purposes of this work. The FPGA provides flexibility, for example, full reconfiguration in milli-seconds and permits a complete single chip solution.
\end{abstract}

Keywords: FPGA, Linear frequency modulation, Digital pulse compression.

\section{Introduction}

One fundamental issue in designing a good radar system is its capability to resolve two small targets that are located at long range with very small separation between them. This requires a radar system to transmit a long pulse which will have enough energy to detect a small target at long range. However, a long pulse degrades range resolution. Hence, frequency or phase modulation of the signal is used to achieve a high range resolution when a long pulse is required. The capabilities of short-pulse and high range resolution radar are significant. For example, high range resolution allows resolving more than one target with good accuracy in range without using angle information. Many other applications of short-pulse and high range resolution radar are clutter reduction, glint reduction, multipath resolution, target classification, and Doppler tolerance.
There are several methods of pulse compression that have been used in the past. The most popular of them is linear frequency modulation (LFM) which was invented by R.H. Dickie in 1945. The other pulse compression techniques are Binary phase codes, Polyphase codes, Barker codes, Costas codes, Nonlinear Frequency Modulation etc. In this paper, we shall implement the LFM pulse compression technique without components around zero frequency.

\section{Pulse Compression Concepts}

A correlation operation at the radar receiver indicates the presence of a received echo by compressing the received signal in time using its time-correlation properties. The correlation operation can also be performed using Matched Filter. In fact, it can be mathematically shown that matched filter using the time reversed complex-conjugate of a signal is equivalent to correlating with the signal. Consider the matched filter operation between a signal $\mathrm{x}(\mathrm{t})$ and an impulse response $\mathrm{h}(\mathrm{t})$ :

$$
y(\tau)=x(t) * h(t)=\int_{-\infty}^{\infty} x(t) h(\tau-t) \cdot d t
$$

The above matched filter operation can be converted into an auto-correlation operation for the signal $x(t)$ by replacing h $(\mathrm{t})$ by $x^{*}(-t)$

$y(\tau)=\int_{-\infty}^{\infty} x(t) x^{*}(\tau-t) \cdot d t=R_{x x}(\tau)$

Thus it is obvious that the auto-correlation operation is mathematically equivalent to matched filter with a timereversed complex conjugate of the signal. In the frequency domain, matched filter can be represented using the product of the Fourier transforms of the signal $\mathrm{x}(\mathrm{t})$ its time-reversed complex-conjugate.

$$
R_{x x}(\tau)=F^{-1}\left\{F[x(t)] F\left[x^{*}(-t)\right]\right\}
$$

For the purposes of simulation and digital implementation, the above operation can also be represented using a discretetime representation. Suppose $\mathrm{x}(\mathrm{t})$ is sampled using a sampling duration $\mathrm{T}_{\mathrm{s}}$ and has a finite number of samples $\mathrm{N}=$ $\mathrm{T} / \mathrm{T}_{\mathrm{s}}$, the auto-correlation sequence can be obtained as:

$$
R_{x x}(m)=\sum_{n=-\infty}^{\infty} x(n) * x^{*}(n-m) \quad \begin{gathered}
\mathrm{m}=0,1, \ldots . . \\
\mathrm{N}-1
\end{gathered}
$$


Since the auto-correlation sequence is symmetric, it is sufficient to consider only the positive lags. The above operation can be implemented using the matched filter operation. By defining $h(n)=x^{*}(-n)$, we have:

$$
\begin{gathered}
R_{x x}(m)=\sum_{n=-\infty}^{\infty} x(n) h(m-n) \\
R_{x x}(m)=x(n) * x^{*}(-n) \\
R_{x x}(m)=\operatorname{IFFT}\left[F F T\{x(n)\} \times F F T\left\{x^{*}(-n)\right\}\right]
\end{gathered}
$$

Where, the FFT and IFFT operations were used to simplify the Correlation operation.

\section{Model of Pulse Compression}

\subsection{LFM Waveform}

LFM signal (also known as the mathematical expression of the Chirp signal):

$$
s(t)=\operatorname{rect}\left(\frac{t}{T}\right) e^{j 2 \pi\left(f_{c} t+\frac{K}{2} t^{2}\right)}
$$

Where $f_{c}$ is the carrier frequency, $\operatorname{rect}\left(\frac{t}{T}\right)$ is the rectangular signal

$$
\operatorname{rect}\left(\frac{t}{T}\right)= \begin{cases}1 & \left|\frac{t}{T}\right| \leq 1 \\ 0 & \text { elsewher }\end{cases}
$$

The LFM waveforms are digitally produced when PROMs are addressed by counters initialized by instructions from the radar control process software. The LFM waveforms, consisting of I and Q values, are D/A converted and applied to transmit IF generation circuits. Twelve parallel bits representing the count are output from the counter function and presented to a phase PROM and a timing PROM. The timing PROM is used to determine the start and end of the transmit gate and the waveform generator cutting gate. Waveform generators no. 1 and 2 transmit gates are ORed to provide a complete gate for the duration of the final transmit waveform used by the exciter. The timing PROM provides countdown/up and stop information to the counter. Initially the counter counts down until a zero count is reached. Then the timing PROM changes the counter instruction to count up. An up count continues until the timing PROM sends a stop command.

The phase PROM has been programmed to produce the addresses for the sine/cosine PROMs upon receipt of the counter output. A 4:1 multiplexer selects one of four sine/cosine sources that allow the waveform generator to produce LFM waveforms.

The sine/cosine PROMs contain sine and cosine tables that produce digitally preprogrammed sine and cosine values. The PROM tables contain the sine and cosine values in offset binary code. Every 100 nanoseconds (10-MHz clock cycle), PROM data is read out. This yields a minimum of 8 points per cycle for the highest pulse frequency. The multiplexer selects the counter output for application to the sine/cosine PROMs. This address controls the sine/cosine PROM readout to provide a $\mathrm{CW}$ signal at the frequency selected that is represented by I and Q output. Fixed maximum or zero outputs may also be selected. The digital LFM waveform outputs, I from the cosine PROM and Q from the sine PROM, are applied to the D/A converters. The D/A converters produce the analog LFM cosine and sine (I and Q) waveforms from the digital outputs of the PROMs.

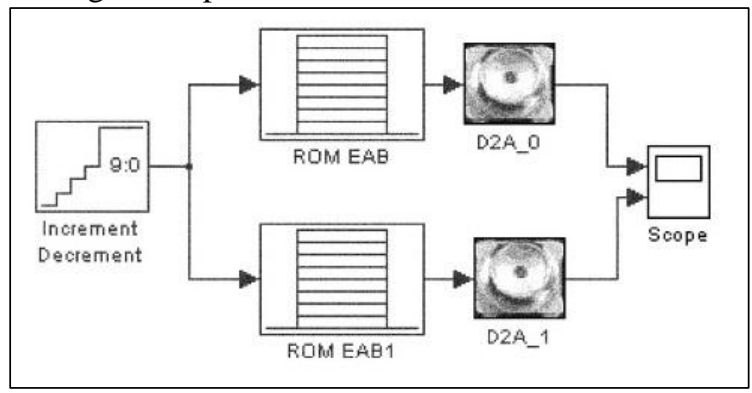

Fig. 1: The Model of Generated Waveform

\subsection{Matched Filter}

The matched filter impulse response of signal $s(t)$ in timedomain should be as follows:

$$
h(t)=s^{*}\left(t_{0}-t\right)
$$

Where $t_{0}$ is the filter additional delay. Theoretical analysis, can make $t_{0}=0$, so, equation(10) could be rewrite as follows:

$$
h(t)=s^{*}(-t)
$$

By substituting from LFM equation into equation (11) we get:

$$
h(t)=\operatorname{rect}\left(\frac{t}{T}\right) e^{-j \pi K t^{2}} \times e^{j 2 \pi f_{c} t}
$$

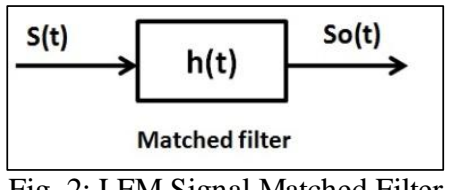

Fig. 2: LFM Signal Matched Filter

As shown in figure 2, we get the output signal $s_{0}(t)$ from the input signal $s(t)$ after passing through the matched filter $h(t)$

$$
\begin{gathered}
S_{0}(t)=S(t) * h(t) \\
S_{0}(t)=\int_{-\infty}^{\infty} h(u) S(t-u) d u
\end{gathered}
$$

$S_{0}(t)$

$$
\begin{aligned}
& =\int_{-\infty}^{\infty} e^{-j \pi K u^{2}} \operatorname{rect}\left(\frac{u}{T}\right) e^{j 2 \pi f_{c} u} \\
& \times e^{j \pi K(t-u)^{2}} \operatorname{rect}\left(\frac{t-u}{T}\right) e^{j 2 \pi f_{c}(t-u)} d u
\end{aligned}
$$

When $0 \leq t \leq T$

$$
\begin{gathered}
S_{0}(t)=\int_{t-T / 2}^{T / 2} e^{j \pi K t^{2}} e^{-j 2 \pi K t u} d u \\
S_{0}(t)=\left.e^{j \pi K t^{2}} \frac{e^{-j 2 \pi K t u}}{-j 2 \pi K t}\right|_{t-T / 2} ^{T / 2} \times e^{j 2 \pi f_{c} t} \\
S_{0}(t)=\frac{\sin \pi K(T-t) t}{\pi K t} e^{j 2 \pi f_{c} t}
\end{gathered}
$$


And when $-T \leq t \leq 0$

$$
S_{0}(t)=\frac{\sin \pi K(T+t) t}{\pi K t} e^{j 2 \pi f_{c} t}
$$

By merge equation (16) and equation (19), we get that:

$$
S_{0}(t)=T \frac{\sin \pi K\left(1-\frac{|t|}{T}\right) t}{\pi K T t} \operatorname{rect}\left(\frac{t}{2 T}\right) e^{j 2 \pi f_{c} t}
$$

Equation (20) is the output impulse response of the LFM matched filter, where the constant carrier frequency is $f_{c}$, and when $t \leq T$ the envelope approximately (sinc) function.

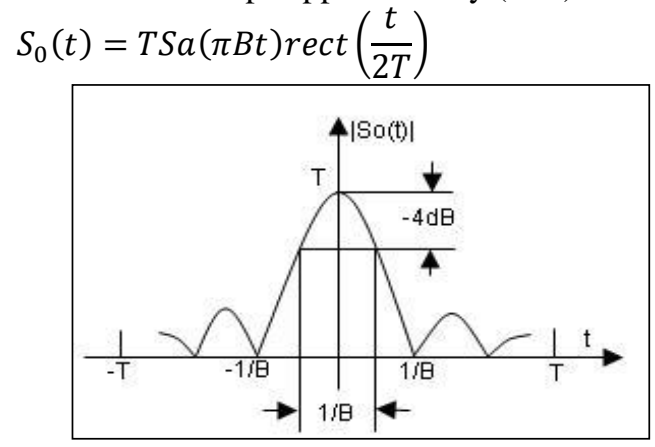

Fig. 3: The Matched Filter Output Signal

Figure 4 shows the model for the pulse compression matched filter. We used in our model for pulse compression four FIR filters with real and imaginary parts at the input. The signal reference is also presented by real and imaginary parts. The real part of pulse compression is calculated by adding the outputs of FIR-1 and FIR-2, and the imaginary part by adding FIR-3 and FIR-4 outputs. Finally, the absolute value (ABS) of the complex signal is calculated applying the ABS CORDIC algorithm, which lets get the pulse compression.

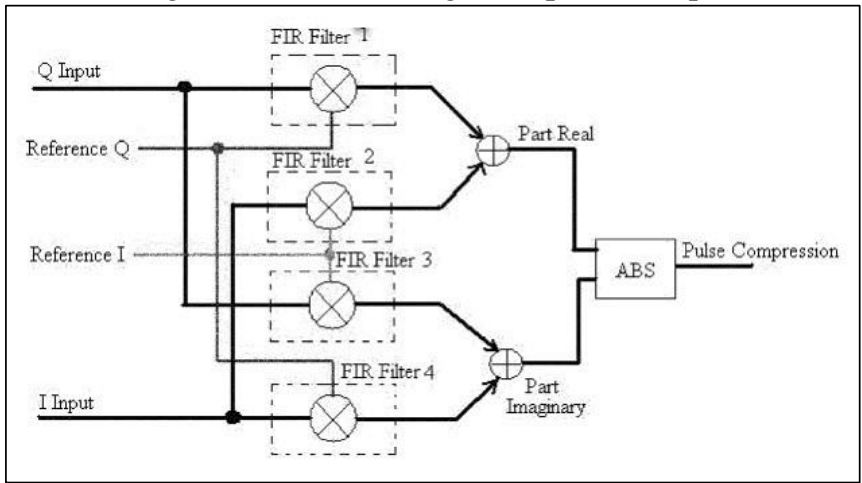

Fig. 4: The Model for Pulse Compression Matched Filter

\section{Matlab Model Results}

In this paper, we take the pulse width is $102.4 \mu$ s, the phase curve is a quadratic curve start from $64 \pi$ to $4 \pi$ at the $49 \mu \mathrm{s}$ then it change linearly to zero at the middle of the pulse then goes up linearly to $4 \pi$ at $53.4 \mu$ s then back again quadratic to $64 \pi$ at the end of pulse. This choice to overcome the zero frequency in the middle of the pulse to avoid jamming and produce a rapid change in frequency which leads to ease tracking of ballistic missiles. Figure 5 \& figure 6 show the phase and frequency against time of the pulse. Figure 7 shows the real and imaginary part of signal. Figure 8 shows the compressed signal after the matched filter.

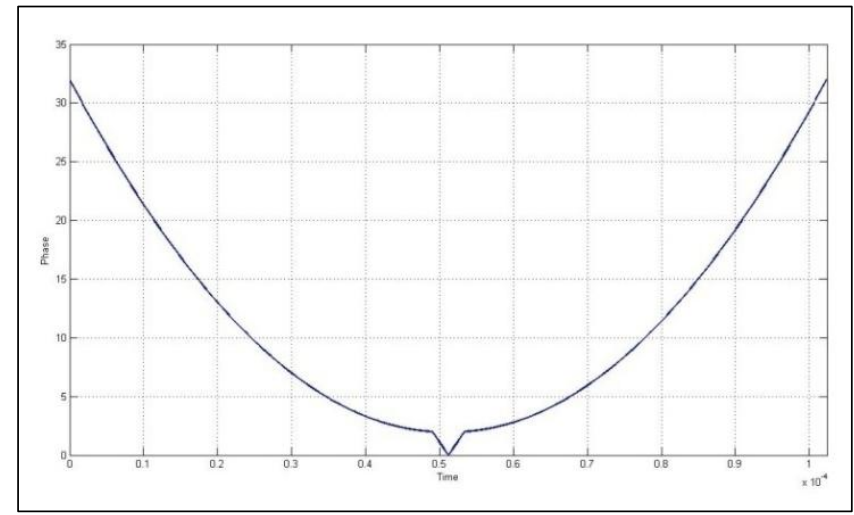

Fig. 5: Phase vs Time

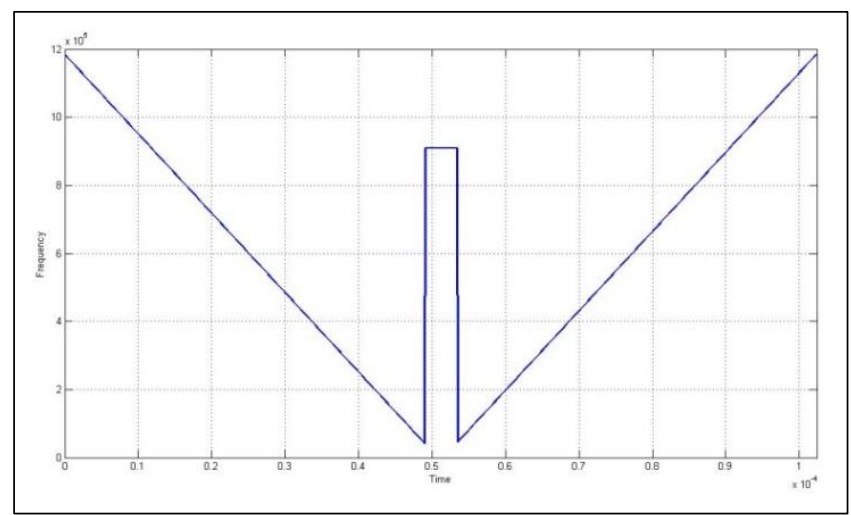

Fig. 6: Frequency vs Time

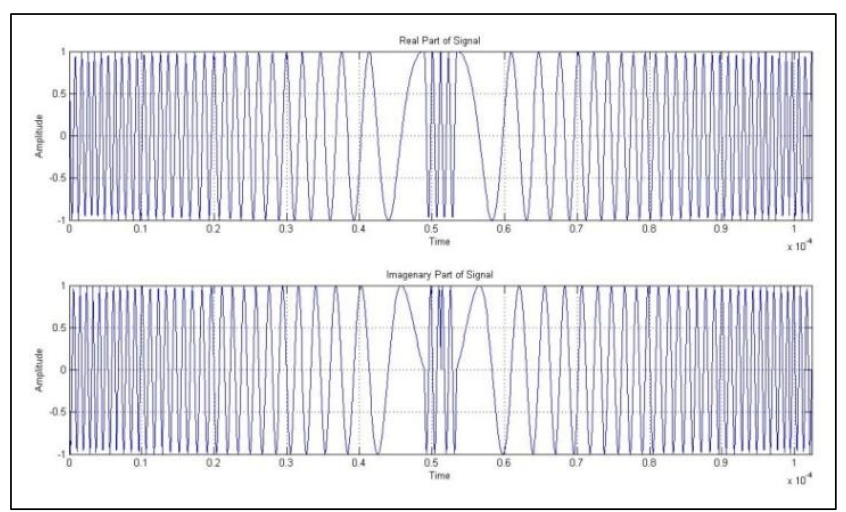

Fig. 7: Real \& Imaginary Parts of Signal

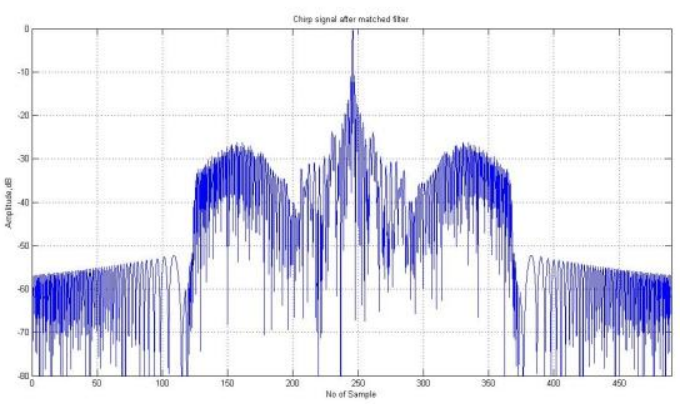

Fig. 8: Signal after Matched Filter 


\section{Hardware Implementation}

High gate count and switching speed of modern FPGA is enabling high data-rate DSP processing to be performed without resorting to ASIC technology. Static RAM based FPGA also enable solutions to be reprogrammable. Then the soft solutions offer flexibility, which is an important attribute of a modem radar system. Consequently, FPGA implementations are attractive in applications where their relatively high unit cost and power consumption are not critical FPGA is adopted here due to the parallel architecture and flexibility to implement. The framework for closed-loop test is shown in fig.3. The synchronous controller, the CORDIC, the decimator and the FFT are implemented on the FPGA.

It has been tested in this work the performance of the FPGAs to generate the radar signal and realize the pulse radar compression. We used the radar data with parameters discussed in models. We employed the Kit Altera FPGA to realize the radar pulse operation.

The model applied to generate the pulse is shown in Fig. 4. Two ROM blocks are used to form the values for real and imaginary parts of a pulse. A cycle counter is applied to represent the address in the ROMs.

The matched filter implementation on FPGA let us eliminating special chips previously needed. We have tested the performance of such a model in the FPGA Altera model CYCLONE-II EP2C8Q208C8N.

The main advantages of proposed structure (the software and FPGA hardware) is the facility whereupon we can easily change the parameters of each a block of the system. It is required in some applications to change the parameters of the generated signal, increase the precision of the system, and then increasing number of bits can be realized in each used block, that let us flexibility to adjust the parameters of the system, and therefore better solutions.

The realization of the matched filter as it is shown in Fig. 4, and the number of taps in each FIR filter was 1024.

\section{Results}

Experimental results measured from the implemented radar processing model using the FPGA Altera model CYCLONEII EP2C8Q208C8N and Quartus II as a software design tool, simulation and logic analyzer tool, we get the results from logic analyzer as following:

\subsection{LFM Waveform Generator}

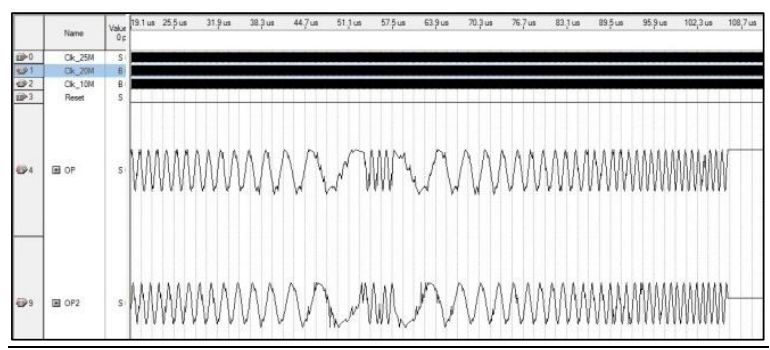

Fig. 9: Generation of Model Waveform

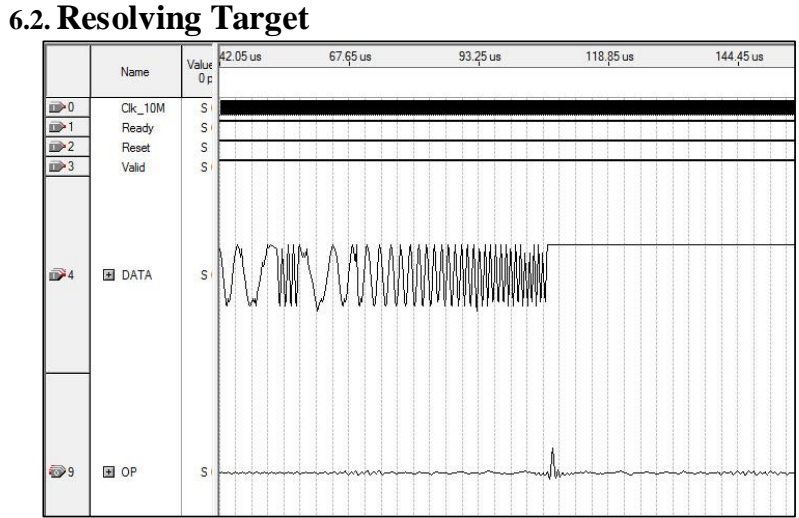

Fig. 10: Resolving One Target

\section{Conclusion}

An efficient very large integrated circuit (VLIC) architecture for making exhaustive search for the identification of the best pulse compression was implemented for using in radar and communication systems for significantly improving the system performance.

A flexible real time implementation of digital pulse compression suitable for all-digital radar receiver architecture. Experimental results obtained presented a very good agreement with the theory. The developed system can further be enhanced to be used for surveillance pulsed radars. The realization based on the FPGA chips makes it easy to change the radar working parameters to adapt the design to different situations which following the concept of software defined radio (SDR).

The proposed architecture is a single chip solution for both generation and receiver processing of pulse compression.

Future research work requires additional investigation in trying to design multi codes in one pulse compression system to overcome the ambiguity.

\section{References}

[1] FanWang, Huotao Gao, Lin Zhou, Qingchen Zhou, Jie Shi, Yuxiang Sun, "Design and FPGA implementation of digital pulse compression for HF chirp radar based on modified orthogonal transformation", IEICE Electronics Express, Vol.8, P1736-1742, October-25-2011.

[2]Zhisheng Yan, Biyang Wen, Caijun Wang, Chong Zhang, "Design and FPGA implementation of digital pulse compression for chirp based on CPRDIC", IEICE Electronics Express, Vol.6, P 780-786, June-10-2009.

[3] J. Lee Blanton,"CUED Medium air-to-air radar using stretch range compression", IEEE 1996 National Radar Conference, Ann Arboe, Michigan, 13-16 May 1996.

[4] Saqib Ejaz, Muhammad Amir Shafiq, Dr. Muhammad Junaid Mughal, "Real time implementation of digital LFM pulse compression technique over acoustic waveguides", International Journal of Engineering \& Technology, Vol.10, No.4.

[5] Enrique Escamilla-Hernandez, Victor Kravchenko, Volodymyr Ponomaryov, Daniel Robles-Camarillo, Luis E. Ramos, “ Real time signal compression in radar using FPGA", Cientifica, Vol.12, Num.3, P 131-138, September 2008.

[6] Merril I. Skolnik, "Introduction to radar systems", $3^{\text {rd }}$ edition, McGrawHill, 2001.

[7] Merril I. Skolnik, "Radar Handbook", $2^{\text {nd }}$ Edition, McGraw-Hill, 1990.

[8] M. Vamsi Krishna, K. Ravi Kumar, K. Suresh, V. Rejesh, "Radar Pulse compression", International Journal of electronics \& Communication technology, Vol.2, SP-1, December 2011. 
[9] Mark-Anthony Govoni, "Linear Frequency Modulation of Stochastic Radar Waveform", Submitted to the Faculty of the Stevens Institute of Technology in partial fulfillment of the requirements for the degree of DOCTOR OF PHILOSOPHY, 2011.

[10] Arojit Roychowdhury, "FIR Filter Design Techniques", M. Tech. credit seminar report, Electronic Systems Group, EE Dept, IIT Bombay, November 2002.

[11] V. A. Pogribnoi, T. Leshchinski, I. V. Rozhankovskii, "Methods of enhancing the compression of short LFM signals", Radioelectronics and communications systems, Vol.51, N0.3, P 143-149, 2008.

[12] N. Balaji, M. Srinivasa Rao, K. Subba Rao, S. P. Singh, N. Madhu Sudhana Reddy, "FPGA Implementation of ternary pulse compression sequences", Proceesings of the international multi conference of engineers and computer scientists 2008, Vol.1, 19-21 March 2008 (Hong Kong).

[13] Mark A. Richards, "Time and Frequency Domain Windowing OF LFM Pulses", 29 September 2006.

[14] Wang Peng, Meng Huadong, Wang Xiqin, "Suppressing Autocorrelation Sidelobes of LFM pulse trains with genetic algorithm", Tsinghua Science And Technology, Vol.13, No.6, December 2008.

[15] Bassem R. Mahafza, "Radar Systems Analysis and Design Using MATLAB", chapman \& Hall/CRC, 2000.

[16] Ashok S. Mudukutore, V. Chandrasekar, R. Jeffrey Keeler, "Pulse Compression for Weather Radars", IEEE Transactions on geosciences and remote sensing, Vol.36, No.1, January 1998.

[17] Masanori Shinriki, Hironori Susaki, "Pulse Compression for a Simple Pulse", IEEE Transactions on aerospace and electronic systems, Vol.44, No.4, P.1623-1629, October 2008.

[18] Fun-Bin Duh, Chia-Feng Juang, Chin-Teng Lin, “ Aneural Fuzzy Network Approach to Radar Pulse Compression”, IEEE Geoscience and remote sensing letters, Vol.1, No.1, January 2004. 